\title{
EFEITO DO CAMINHO DE SOLIDIFICAÇÃO NA MICROESTRUTURA E NA PRECIPITAÇÃO DE (TI,NB)(C,N) EM UM AÇO HTP COM BAIXO MN-ALTO NB *
}

\author{
Diana Pérez Escobar ${ }^{1}$ \\ Cynthia Serra Batista Castro ${ }^{1}$ \\ Elenice Cavichioli Borba ${ }^{1}$ \\ Alisson Paulo de Oliveira² \\ Kenji Camei ${ }^{3}$ \\ Ed Juarez Mendes Taiss ${ }^{4}$ \\ André Luiz Vasconcellos da Costa e Silva ${ }^{5}$ \\ Margareth Spangler Andrade ${ }^{1}$
}

\section{Resumo}

O efeito do caminho de solidificação de um aço High Temperature Processing (HTP) API 5L X65 com 0,09 \%Nb, 0,039\%C e 0,54\% Mn (percentagem em massa) na segregação, microestrutura e distribuição dos precipitados de $\mathrm{Ti}, \mathrm{Nb}(\mathrm{C}, \mathrm{N})$ foi estudado por microscopia ótica, eletrônica de varredura (MEV) e simulação computacional (ThermoCalc e DICTRA). Os resultados foram comparados com os de um aço microligado comercial com 0,04 \%Nb, 0,09\% e 0,97 \%Mn. Foi encontrado que o principal parâmetro que influencia no caminho de solidificação é o teor de $\mathrm{C}$, o que está de acordo com a maior micro e macrossegregação observada no aço microligado comercial, em comparação ao aço HTP.

Palavras-chave: Caminho de solidificação; Segregação, Aços microligados, Precipitação de $(\mathrm{Ti}, \mathrm{Nb})(\mathrm{C}, \mathrm{N})$.

\section{EFFECT OF THE SOLIDIFICATION PATH ON THE MICROSTRUTURE AND $(T I, N B)(C, N)$ PRECIPITATION ON A HTP WITH LOW MN HIGH NB STEEL}

\section{Abstract}

The effect of the solidification path of a High Temperature Processing (HTP) steel API $5 \mathrm{~L}$ X65 with $0,09 \% \mathrm{Nb}, 0,039 \% \mathrm{C}$ and $0,54 \%$ of $\mathrm{Mn}$ (weight percent) on the segregation, as-cast microstructure and precipitation distribution of $\mathrm{Ti}, \mathrm{Nb}(\mathrm{C}, \mathrm{N})$ was studied by means of optical, scanning electron microscopy (SEM) and computational simulation (ThermoCalc and DICTRA). The results were compared with those obtained for a commercial microalloyed steel, containing $0.04 \% \mathrm{Nb}, 0,09 \% \mathrm{C}$ and $0.97 \% \mathrm{Mn}$. The results indicate that the main parameter that influences in the solidification path is the $\mathrm{C}$ content, which concurs with the higher micro and macrosegregation observed for the commercial microalloyed steel, compared to the HTP steel.

Keywords: Solidification path, Segregation, Microalloyed steels, $(\mathrm{Ti}, \mathrm{Nb})(\mathrm{C}, \mathrm{N})$ precipitation.

1 Doutora, Pesquisadora em Tecnologia, Instituto SENAl de Inovação em Metalurgia e Ligas Especiais, CIT SENAI FIEMG, Belo Horizonte, MG, Brasil.

2 Engenharia Metalúrgica, M.Sc.,Assessor Técnico, Gerdau Ouro Branco, Ouro Branco, MG, Brasil.

3 Engenharia Metalúrgica, Ph.D, Gerente de P\&D, Chapas grossas, Gerdau Ouro Branco, Ouro Branco, MG, Brasil.

4 Engenharia Metalúrgica, Consultor, Companhia Brasileira em Mineração e Metalurgia CBMM, Araxá, MG, Brasil.

5 Engenharia Metalúrgica, Ph.D., Professor Titular, EEIMVR-UFF, Volta Redonda, RJ, Brasil. 


\section{INTRODUÇÃO}

Durante a solidificação dos aços, ocorre a partição de solutos entre o líquido e os cristais formados na fase sólida. Este fenômeno, chamado de segregação, produz uma distribuição heterogênea dos elementos químicos no sólido, a qual permanece durante o processamento e no produto final, sob a forma de microssegregação [1]. Dependendo dos movimentos do líquido durante a solidificação pode ocorrer, também, a macrossegregação [2-4], que pode alcançar escalas próximas da dimensão da placa. Enquanto a microssegregação pode ser reduzida por meio de longos tratamentos térmicos, que são economicamente inviáveis é impossível eliminar a macrossegregação, que causa variação da microestrutura e das propriedades mecânicas ao longo do material [5].

Chapas grossas de alta resistência usualmente apresentam altos teores de $\mathrm{Mn}$, o que causa efeitos indesejados de segregação durante a solidificação. Este efeito pode ser observado pela distribuição das inclusões de MnS [6] que afeta as propriedades mecânicas e a resistência à fragilização por hidrogênio do produto final. Nióbio, titânio e vanádio, são adicionados aos aços para melhorar o refinamento do grão durante o processamento no campo austenítico e para contribuir no endurecimento por precipitação [7]. O Nióbio é conhecido por melhorar significativamente as propriedades mecânicas dos aços alta resistência-baixa liga (ARBL), mesmo em teores baixos [8-10], e por retardar a recristalização impedindo o crescimento de grão (entre 1000 e $1300^{\circ} \mathrm{C}$ ). $\mathrm{O}$ enriquecimento do líquido interdendrítico é responsável pela distribuição heterogênea dos solutos no aço, podendo levar a formação de precipitados primários de carbonitretos de titânio e de nióbio $(>1 \mu \mathrm{m})$ [11] que não se dissolvem durante a etapa de reaquecimentos de placas, causando uma diminuição dos elementos microligantes em solução. Além disto, devido ao seu tamanho, pode ocorrer um aumento das tensões locais que pode levar a formação de trincas. Outro efeito negativo da segregação é a distribuição heterogênea dos precipitados menores $(<1 \mu \mathrm{m})$ o que pode diminuir, localmente, o efeito do refinamento de grão, causar crescimento anormal e levar a uma distribuição bimodal do tamanho de grão, causando variações nas propriedades mecânicas ao longo da placa [12].

A redução do $M n$, abaixo de $1,2 \%$ (em massa), $C$ e $P$ é um dos mais recentes desenvolvimentos na produção de aços microligados [13], a qual procura minimizar o bandeamento e a segregação na linha central durante o lingotamento continuo. A diminuição no limite de escoamento, devido à redução de $\mathrm{Mn}$, pode ser compensada pela adição de $\mathrm{Cr}$ e $\mathrm{Nb}$. Um maior teor de $\mathrm{Nb}$ ira melhorar a resposta termomecânica da austenita durante o processamento e altas temperaturas, conhecido como High Temperature Processing - HTP [13-14]

Diferentes modelos computacionais têm sido utilizados para estudar a redistribuição de solutos. O modelo mais simples, proposto por Scheil [15], assume que o líquido é sempre homogêneo e que o sólido formado não se homogeneíza. Atualmente, com os avanços em computação, existem softwares comerciais, que conjugados a bancos de dados termodinâmicos e de mobilidade (ex. DICTRA), permitem a realização de cálculos que consideram a difusão no sólido e no líquido, podendo obter resultados mais realísticos que os obtidos pelo modelo do Scheil [16].

Mesmo com uma extensa literatura em segregação, é importante entender os efeitos da composição química no caminho de solidificação e sua influência na segregação em diferentes posições da placa. É também de interesse industrial entender a influência dos parâmetros de solidificação na morfologia, composição química e 
distribuição dos precipitados de $(\mathrm{Nb}, \mathrm{Ti})(\mathrm{C}, \mathrm{N})$, a qual afeta a solubilização do $\mathrm{Nb}$ e a sua precipitação nas etapas posteriores de produção.

O presente trabalho tem por objetivo correlacionar o caminho de solidificação com a segregação, a microestrutura solidificada e a distribuição dos precipitados de $(\mathrm{Ti}, \mathrm{Nb})(\mathrm{C}, \mathrm{N})$ em dois aços microligados em diferentes posições nas placas de lingotamento contínuo, visando contribuir ao projeto das ligas e de seu processamento. Assim, dois aços, um com 0,039\% $0.09 \% \mathrm{Nb}$ e $0.54 \%$ e $\mathrm{Mn}$ e outro aço com $0,09 \%$ C $0.04 \% \mathrm{Nb}$ e $0.97 \% \mathrm{Mn}$ foram estudadas empregando microscopia ótica e confocal e microscopia eletrônica de varredura. Os resultados foram comparados com os obtidos por simulação computacional (ThermoCalc e DICTRA).

\section{MATERIAIS E MÉTODOS}

A composição química dos dois aços está apresentada na Tabela 1. Ambos os materiais foram recebidos na condição bruta de lingotamento. O aço HTP API 5L X65 Sour foi projetado pela CBMM (Companhia Brasileira de Metalurgia e Mineração) e o aço com teores de $\mathrm{C}$ e $\mathrm{Mn}$ mais elevados e $\mathrm{Nb}$ mais baixo, que será denominado a partir de agora como "CMn_Baixo Nb", foi produzido pela Gerdau Ouro Branco.

Tabela 1. Composição química dos aços HTP API 5L X65 e CMn_Baixo Nb (todas as composições, exceto se indicado de outra forma, são em \% em massa).

\begin{tabular}{ccccccc}
\hline Aço & C & Mn & Nb & Si & Al & Adições realizadas \\
\hline HTP API 5L X65 & 0.039 & 0.54 & 0.090 & 0.21 & 0.029 & $\mathrm{Cu}, \mathrm{Ni}, \mathrm{Ti}, \mathrm{Cr}$ e N \\
\hline CMn_Baixo Nb & 0.09 & 0.97 & 0.038 & 0.26 & 0.025 & $\mathrm{Cu}, \mathrm{Ni}, \mathrm{Ti}, \mathrm{Cr}$ e N \\
\hline
\end{tabular}

Foi estudada a microestrutura das amostras lingotadas e a distribuição de inclusões e dos precipitados de $(\mathrm{Ti}, \mathrm{Nb})(\mathrm{C}, \mathrm{N})$ em três posições dos aços: região da linha central de segregação (1/2 da espessura), a 1/4 da espessura e no ponto triplo. As amostras foram preparadas por lixamento e polimento até $1 \mu \mathrm{m}$ e atacadas com Nital $2 \%$. As amostras do ponto triplo foram analisadas na seção transversal enquanto que as amostras retiradas a $1 / 2$ e a $1 / 4$ da espessura foram analisadas na seção longitudinal.

A análise dos precipitados localizados nas regiões interdendríticas e intradendríticas em microssegregação foi feita em dois estágios usando microscopia eletrônica de varredura. Inicialmente, foi calculada a fração de área de regiões interdendríticas e intradendríticas. Posteriormente, 3 regiões interdendríticas e 3 regiões intradendríticas, para cada amostra, foram estudadas detalhadamente com um aumento de 20000X. Foram realizadas medições de EDS para cada partícula buscando identificar a presença de $\mathrm{Ti}$ e de $\mathrm{Nb}$. Nas partículas onde o $\mathrm{Nb}$ e $\mathrm{o} \mathrm{Ti}$ estavam presentes, foi calculado o diâmetro médio e a área para posteriormente calcular a fração de área de precipitados nas regiões interdendríticas e intradendríticas.

Para a simulação da segregação no software DICTRA foi usado um modelo que considera uma célula com geometria cilíndrica com raio definido como a metade do espaçamento dendrítico secundário $(\mu / 2)$, Figura 1. Nesta célula, a solidificação começa na superfície externa do cilindro e termina na região central, interdendrítica. Para medição do espaçamento secundário interdendrítico as amostras retiradas a 1/4 da espessura foram atacadas com o reagente dodecilbenzeno sulfonato de sódio [17]. Posteriormente, mapas de Mn (obtidos por Wavelength Dispersive Spectrum- 
WDS) foram feitos, já que o reagente não conseguiu revelar as dendritas do aço HTP API 5L X65.

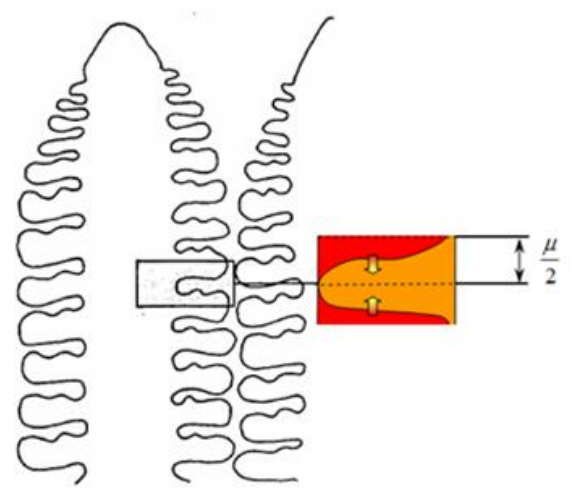

Figura 1. Esquema do espaçamento dendrítico secundário $(\mu)$ e região usada para modelamento da solidificação [18].

É importante considerar que, no modelo de difusão (DICTRA) (a) não existe fluxo de massa entre a região modelada e sua vizinhança. Diferenças de densidade, efeito do "bulging ou soft-reduction", etc., não são simuladas. Ou seja, a simulação da macrossegregação precisa ser feita por outros métodos e (b) somente a difusão é considerada, isto é, não se considera eventual convecção no líquido, que ocorreria nas regiões sujeitas a macrosegregação. Ainda assim o modelo é válido para a maior parte do volume da placa, onde não ocorrem movimentos macroscópicos de liquido durante a solidificação e não há macrosegregação.

\section{RESULTADOS E DISCUSSÃO}

\subsection{Medições do espaçamento interdendrítico}

A figura $2 \mathrm{a}$ e $2 \mathrm{~b}$ apresentam as imagens de microscopia ótica das dendritas obtidas após ataque com dodecilbenzeno sulfonato de sódio. Foi possível observar que as dendritas foram reveladas nitidamente para o aço CMn_Baixo Nb (figura 2b), enquanto que para o aço HTP API 5L X65 não foram observadas dendritas. A figura 2c apresenta o resultado do mapeamento de Mn por WDS do aço HTP API 5L X65, feito no centro de pesquisa da Mannesman Salzgitter.

Os resultados de WDS confirmaram que a solidificação do aço HTP API 5L X65 apresenta uma morfologia celular e que não foram formadas dendritas secundárias, diferentemente do aço CMn_Baixo $\mathrm{Nb}$. Esta diferença na morfologia de solidificação foi explicada por Campbell [19] e está relacionada com uma menor diferença entre as temperaturas liquidus e solidus durante a solidificação, como acontece no caso do HTP API 5L X65, devido ao menor teor de carbono. Esta diferença sugere uma zona rica em soluto menor na frente de solidificação, comparada ao do aço CMn_Baixo $\mathrm{Nb}$, a qual afeta o super-resfriamento constitucional e igualmente a morfologia de solidificação. 


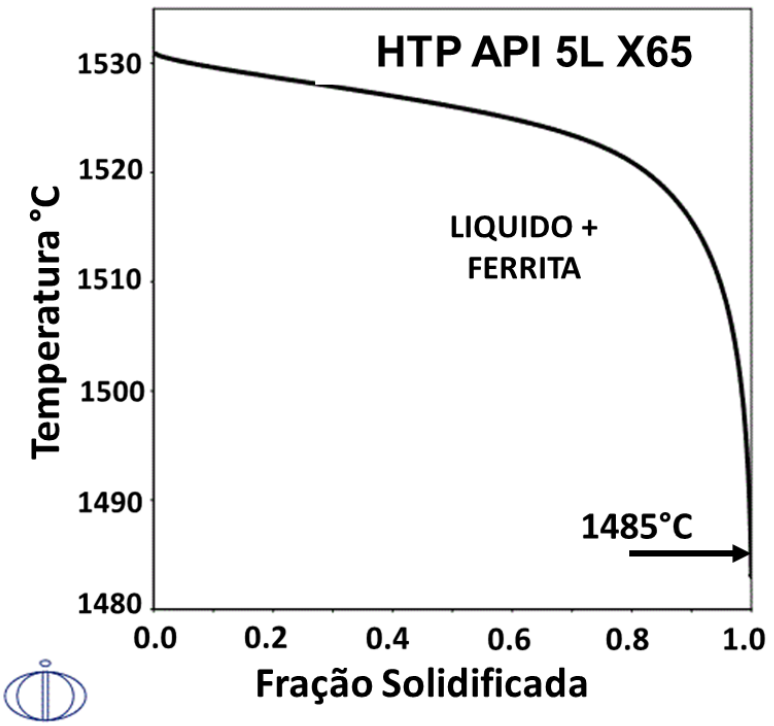

(a)

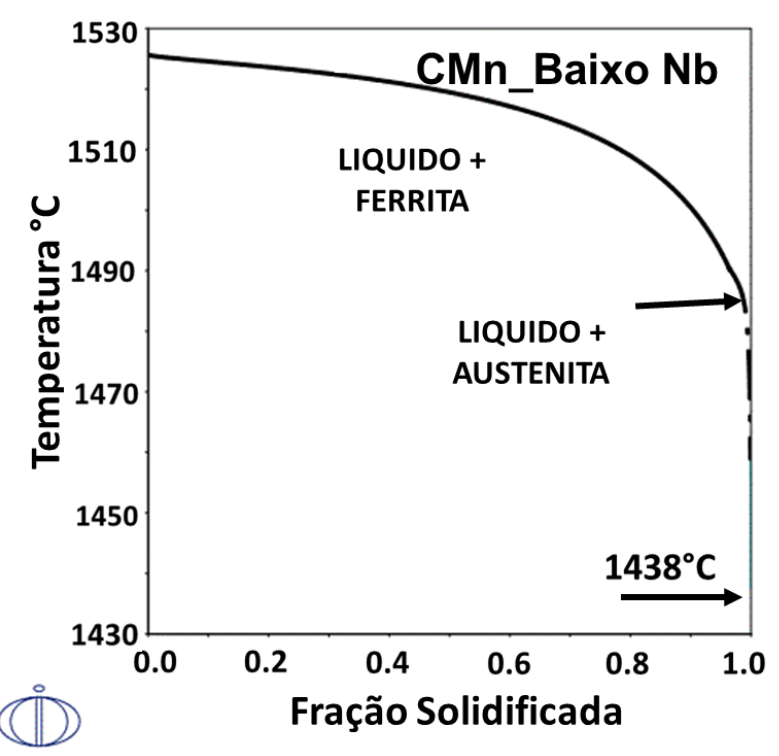

(b)

Figura 3. Simulação da sequência de solidificação, a $1 / 4$ da espessura, calculada com 0 uso do programa DICTRA para os aços (a) HTP API 5L X65 e (b) CMn_Baixo Nb.

A distribuição da concentração de solutos na fase líquida durante a solidificação é apresentada na Figura 4. Estes resultados foram utilizados para calcular a temperatura de precipitação do $(\mathrm{Ti}, \mathrm{Nb})(\mathrm{C}, \mathrm{N})$ no ThermoCalc. Os valores calculados foram de $1513^{\circ} \mathrm{C}$ para o aço HTP API $5 \mathrm{~L}$ X65 e $1478^{\circ} \mathrm{C}$ para o aço CMn_Baixo Nb e estão sinalizados por uma linha pontilhada.
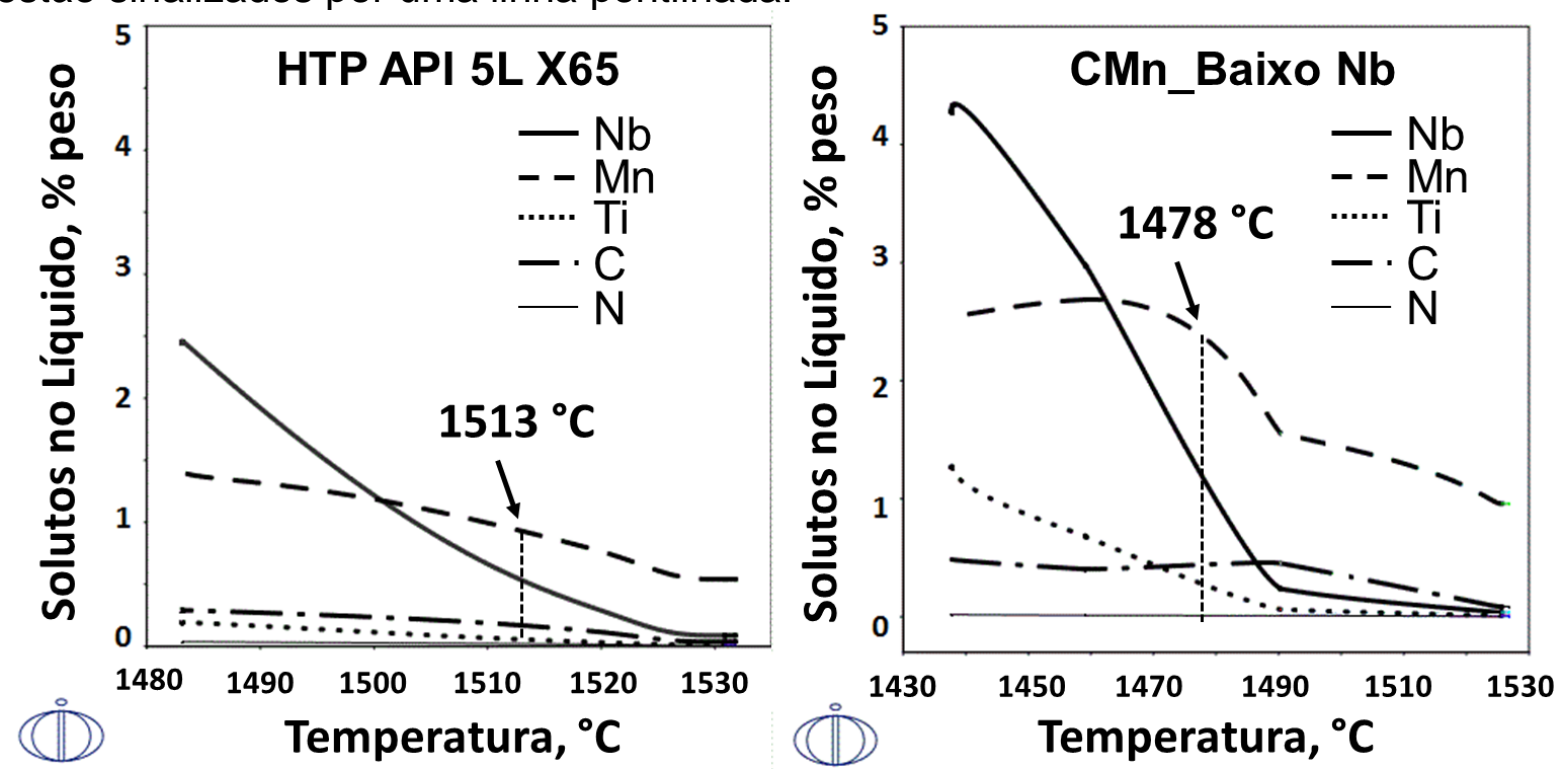

(b)

Figura 4. Simulação obtida com o uso do programa DICTRA da distribuição da concentração de solutos na fase líquida, durante solidificação, para a posição a $1 \frac{1}{4}$ da espessura para os aços (a) HTP API $5 \mathrm{~L}$ X65 e (b) CMn_Baixo Nb. Linhas pontilhadas representam a temperatura de precipitação dos precipitados de $(\mathrm{Ti}, \mathrm{Nb})(\mathrm{C}, \mathrm{N})$ calculados com o uso do programa ThermoCalc de $1513^{\circ} \mathrm{C}$ para o aço HTP API 5L X65 e $1478^{\circ} \mathrm{C}$ para o aço CMn_Baixo Nb.

A partir das temperaturas de precipitação os valores de concentração do Ti, C e N, são superestimados, devido à segregação. Isto ocorre porque o modelo adotado pelo DICTRA não prediz a precipitação do $(\mathrm{Ti}, \mathrm{Nb})(\mathrm{C}, \mathrm{N})$. A microssegregação do $\mathrm{Mn}$ no líquido não é afetada pela precipitação devido ao limite de solubilidade deste elemento no precipitado. Para o aço $\mathrm{CMn} \_$Baixo $\mathrm{Nb}$, que solidifica por 
transformação peritética, o $\mathrm{Mn}, \mathrm{Nb}$ e $\mathrm{Ti}$ apresentam uma forte tendência para segregar podendo alcançar concentrações 10 vezes maior que a nominal nas regiões interdendríticas. Mesmo que o aço HTP API 5L X65 apresente teor mais elevado de $\mathrm{Nb}$ que o $\mathrm{CMn}$ _Baixo $\mathrm{Nb}$, este elemento segrega mais no último devido à diferença dos caminhos de solidificação.

\subsection{Caracterização microestrutural e distribuição dos precipitados de $(\mathrm{Ti}, \mathrm{Nb})(\mathrm{C}, \mathrm{N})$}

Ambos os aços apresentaram microestrutura ferrítica/perlítica, sendo que o aço CMn_Baixo $\mathrm{Nb}$ tem uma maior presença de perlita, especialmente na linha central de segregação. Inclusões de MnS, óxidos e precipitados primários de $(\mathrm{Ti}, \mathrm{Nb})(\mathrm{C}, \mathrm{N})$ (> $1 \mu \mathrm{m}$ ) foram observados nas três posições no aço CMn_Baixo $\mathrm{Nb}$, enquanto os mesmos foram observados, em menor quantidade e tamanho, somente na posição do centro para o aço HTP API 5L X65 (Figura 5). Regiões interdendríticas e intradendríticas em microssegregação (precipitados de $(\mathrm{Ti}, \mathrm{Nb})(\mathrm{C}, \mathrm{N})$ menores que 1 $\mu \mathrm{m})$ foram encontradas em todas as posições nos dois aços [12,22].

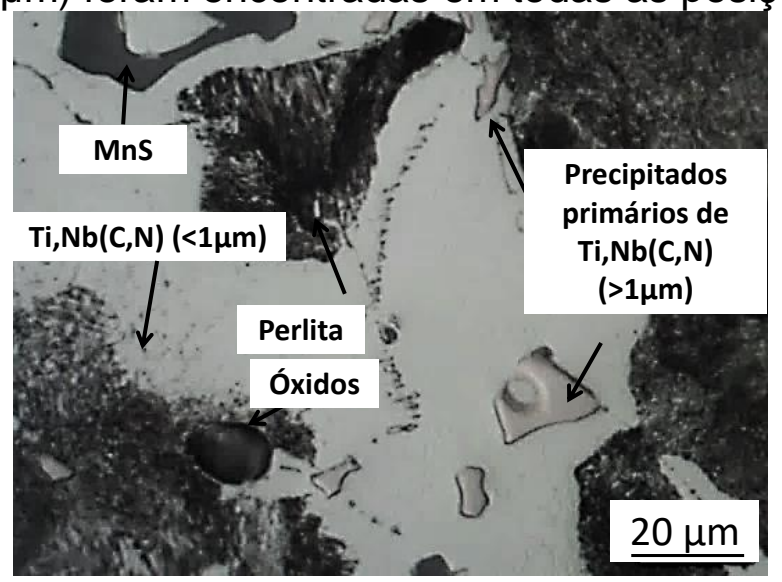

(a)

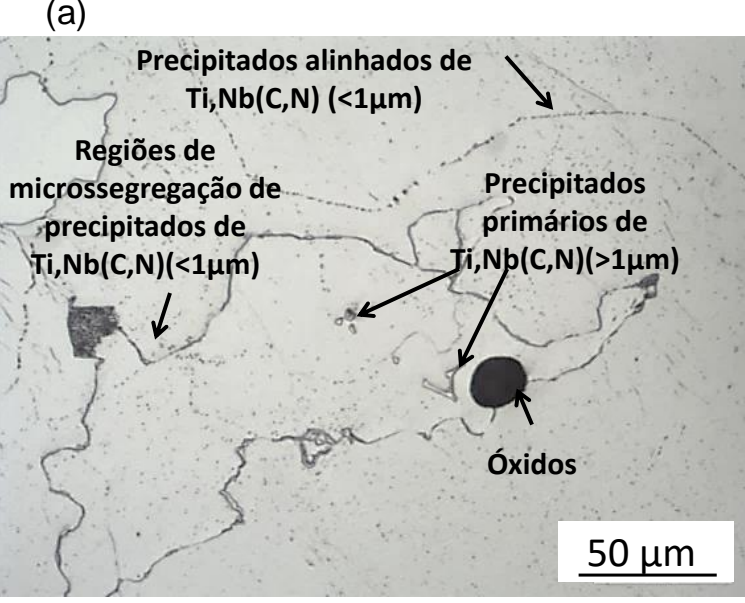

(c)

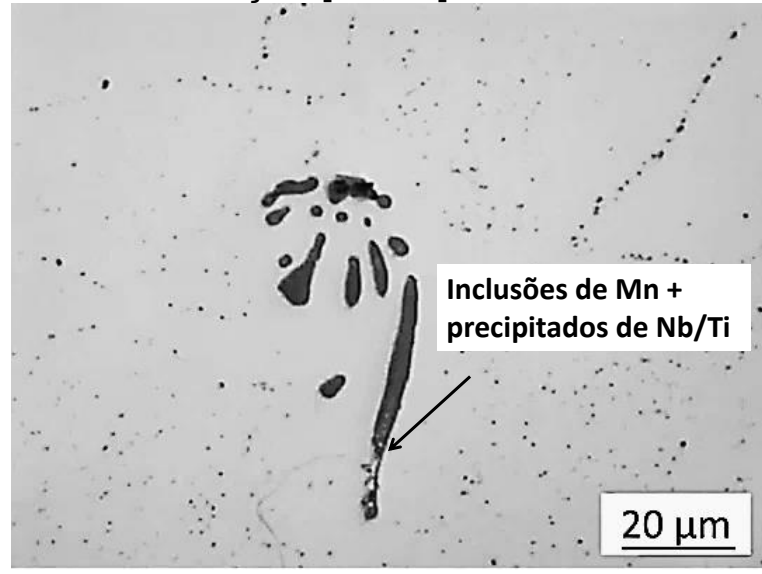

(b)

Figura 5. Imagens de microscopia ótica do aço CMn_Baixo Nb obtidas nas posições a (a) $1 / 2$ da espessura, (b) $1 / 4$ da espessura e (c) Aço HTP API 5 L X65 na posição a $1 / 2$ da espessura. Microestrutura ferrítica/perlítica foi observada. Inclusões e precipitados primários de $(\mathrm{Ti}, \mathrm{Nb})(\mathrm{C}, \mathrm{N})$ foram observados somente na posição a 1/2 da espessura no aço HTP API 5L X65. Regiões interdendríticas com aglomeração de precipitados de $(\mathrm{Ti}, \mathrm{Nb})(\mathrm{C}, \mathrm{N})$, menores que $1 \mu \mathrm{m}$, foram observados em todas as posições para ambos os aços. 
A figura 6 mostra uma das 7 imagens utilizadas para calcular a fração de área de regiões interdendríticas e intradendríticas, para posição, em ambas amostras. Os resultados destas medidas são apresentados na Tabela 3.
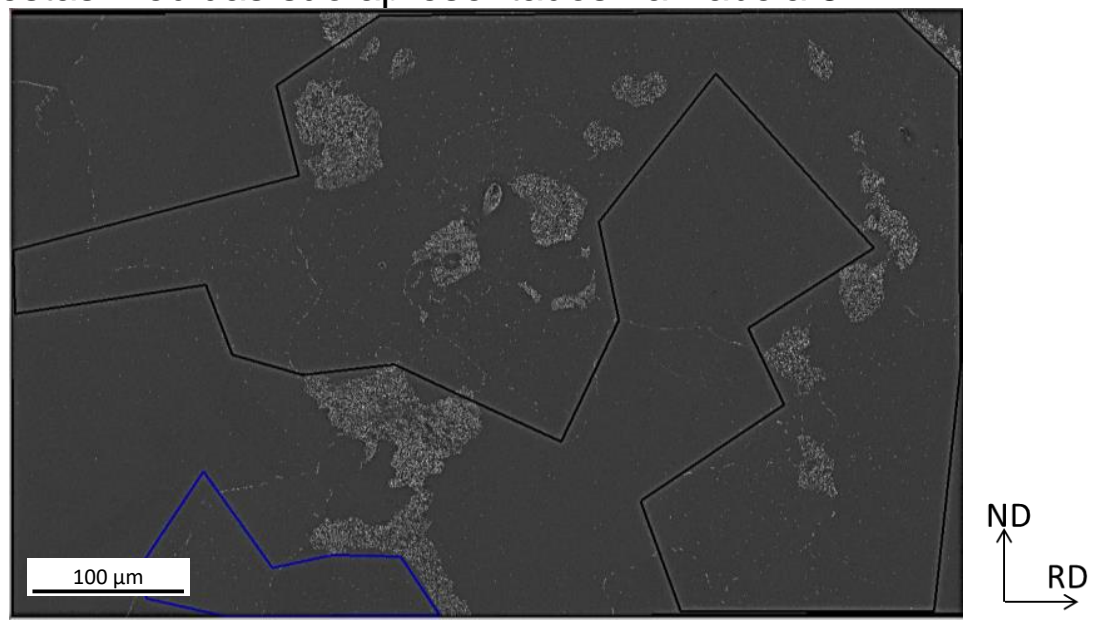

Figure 6. Imagem de microscopia eletrônica de varredura da amostra retida a $1 / 2$ da espessura do aço HTP API $5 \mathrm{~L}$ X65. As regiões em destaque mostram as regiões interdendríticas ricas em precipitados de $(\mathrm{Ti}, \mathrm{Nb})(\mathrm{C}, \mathrm{N})$.

Tabela 3. Fração de área de regiões interdendríticas e intradendríticas

Fração de área de regiões interdendríticas e intradendríticas

\begin{tabular}{ccccc}
\hline Posição / Aço & \multicolumn{2}{c}{ HTP API 5L X65 } & \multicolumn{2}{c}{ CMn_Baixo Nb } \\
\hline Região & Inter & Intra & Inter & Intra \\
\hline 1/2 espessura & 18.8 & 81.2 & 3.8 & 96.2 \\
\hline 1/4 espessura & 2.6 & 97.4 & 3.4 & 96.6 \\
\hline Ponto triplo & 3 & 97 & 3.4 & 96.6 \\
\hline
\end{tabular}

Precipitados com morfologia de estrela (cruciforme) foram encontrados (figura 7), tanto na perlita como na ferrita, em todas as amostras. Foi observado que a morfologia e o tamanho estão relacionados. Os precipitados maiores que $100 \mathrm{~nm}$ são cruciformes, enquanto que nos precipitados com tamanho entre 70 e $100 \mathrm{~nm}$ foi possível observar pequenos braços. Os precipitados menores que $70 \mathrm{~nm}$ têm uma morfologia esférica. Não foram encontrados precipitados cuboides. A figura 8 apresenta os resultados da distribuição de tamanho dos precipitados nas regiões interdendríticas e intradendríticas, para as três posições, em ambos os aços.

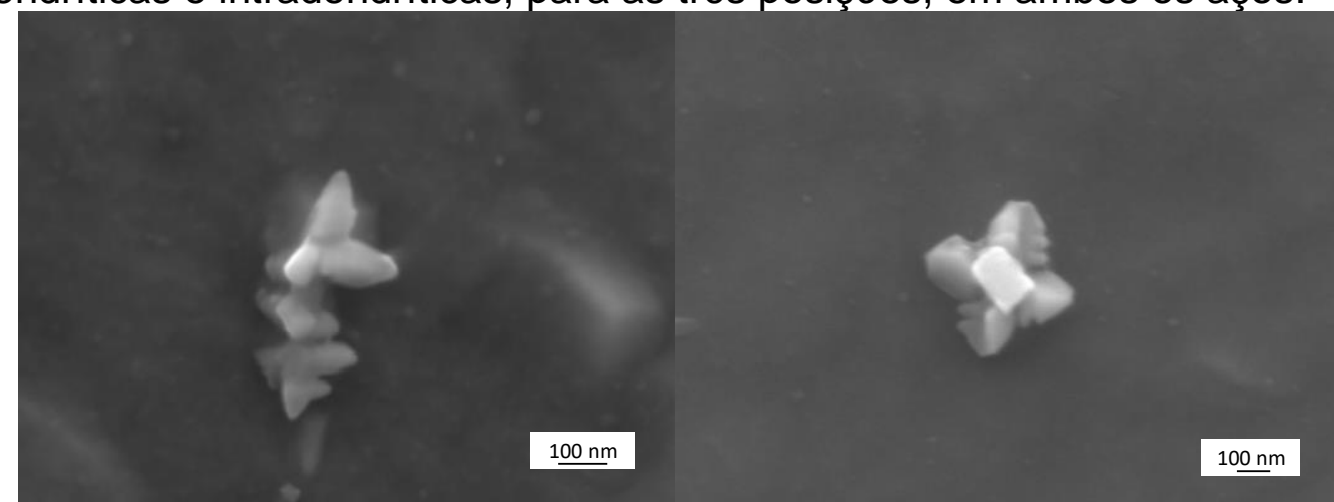

Figure 7. Imagens de MEV dos precipitados cruciformes de (Ti,Nb)(C,N) no aço CMn_Baixo Nb a $1 / 2$ da espessura. Abaixo de $100 \mathrm{~nm}$, a morfologia do precipitado é mais próxima de uma esfera. 


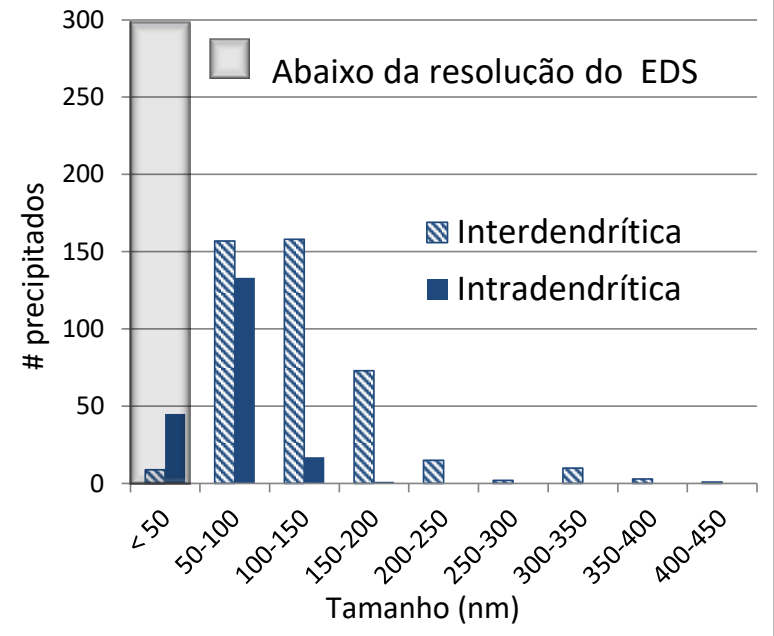

(a)

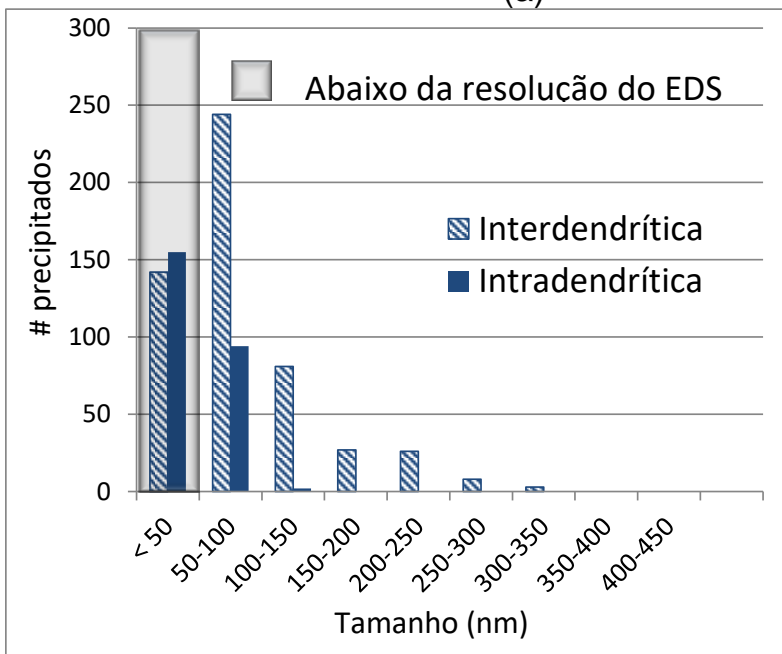

(c)

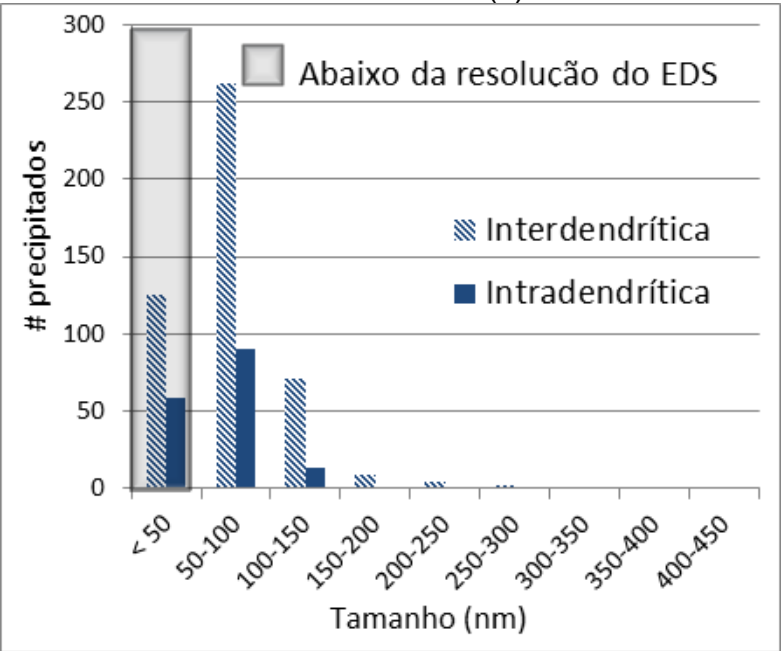

(e)

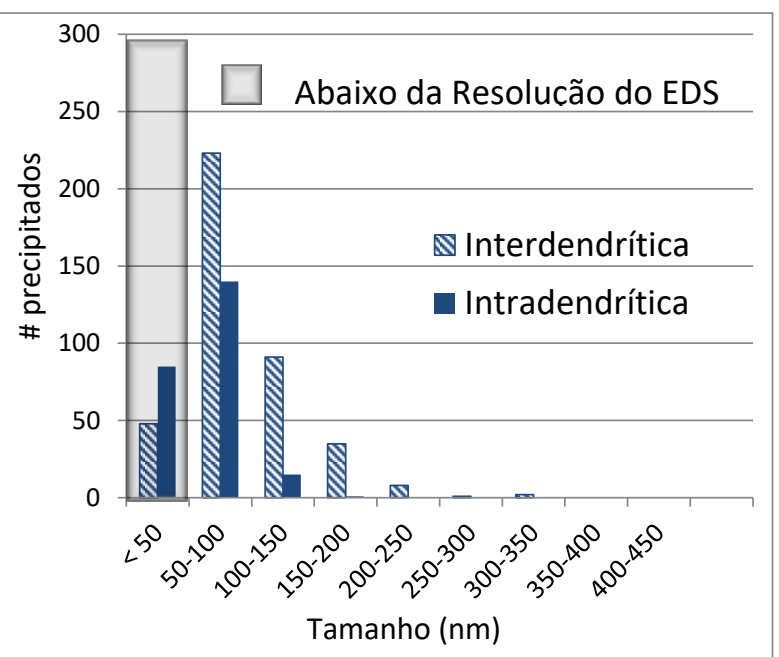

(b)

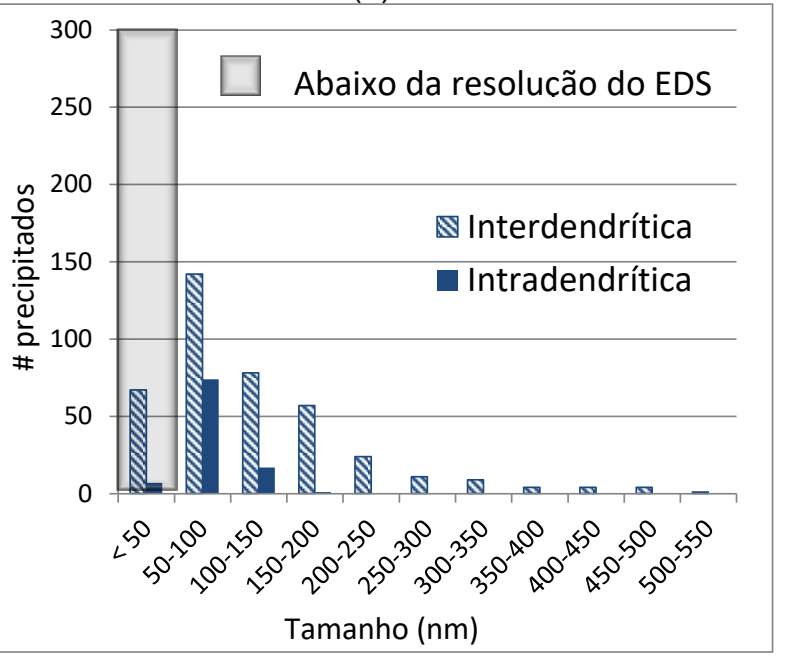

(d)

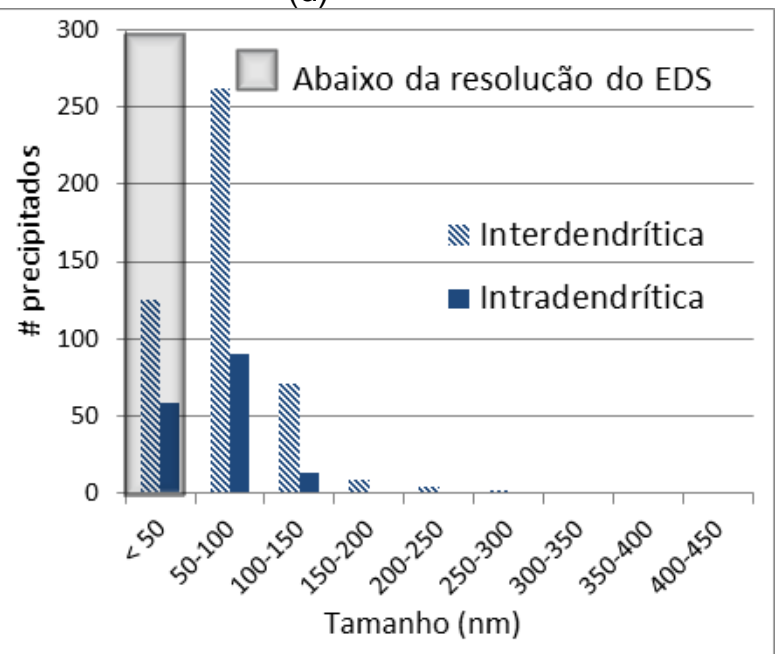

(f)

Figure 8. Distribuição de tamanho dos precipitados de $(\mathrm{Ti}, \mathrm{Nb})(\mathrm{C}, \mathrm{N})$, nas regiões inter e intradendrítica, para (a) CMn_Baixo Nb $1 / 2$ espessura, (b) CMn_Baixo Nb 1/4 espessura, (c) CMn_Baixo $\mathrm{Nb}$ ponto triplo, (d) HTP API $5 \mathrm{~L}$ X65 1/2 espessura, (e) HTP API 5L X65 1/4 espessura e (f) HTP API 5L X65 ponto triplo. A região sombreada nos gráficos $(<50 \mathrm{~nm})$ represente os resultados que estão abaixo da resolução do EDS.

Foi encontrado que o tamanho dos precipitados nas regiões intradendríticas varia de 0 a $200 \mathrm{~nm}$, enquanto que nas regiões interdendríticas podem alcançar um diâmetro 
de até $550 \mathrm{~nm}$. Como esperado, a população de precipitados é maior nas regiões interdendríticas. Para algumas partículas menores que $50 \mathrm{~nm}$ não foram encontrados $\mathrm{Nb}$ nem $\mathrm{Ti}$, mesmo elas tendo morfologia similar à dos precipitados. As mesmas não foram incluídas na distribuição e por esta razão o valor da frequência para os precipitados entre 0 e 50 foi sombreada, sinalizando resultados abaixo da resolução do EDS. Roy [22] encontrou que a frequência de precipitados com diâmetro menor a $50 \mathrm{~nm}$ é muito maior que a dos precipitados maiores de $50 \mathrm{~nm}$ e que existe uma relação exponencial da frequência com o inverso do tamanho dos precipitados. Os valores da fração de área dos precipitados nas regiões interdendríticas e intradendríticas estão apresentados na Tabela 4.

Tabela 4. Fração de área dos precipitados por região, para cada posição, em ambos os aços.

\begin{tabular}{ccccc}
\hline \multicolumn{3}{c}{ Fração de área dos Precipitados (x10E-04) } \\
\hline Posição / Aço & \multicolumn{1}{c}{ HTP API } & L X65 & \multicolumn{2}{c}{ CMn_Baixo Nb } \\
\hline Região & Inter & Intra & Inter & Intra \\
\hline 1/2 espessura & 91.8 & 6.9 & 79.5 & 9.3 \\
1 1/4 espessura & 47.2 & 7.1 & 42.5 & 1.0 \\
Ponto triplo & 33.6 & 7.0 & 56.4 & 6.5 \\
\hline
\end{tabular}

Os resultados mostram uma fração de área de precipitados 10 vezes maior nas regiões interdendríticas, o que está de acordo com a literatura [22] e mostra o efeito da microssegregação. Além disso, a fração de área é maior na região a $1 / 2$ da espessura, para ambos os aços, e estaria relacionado com 0 efeito da macrossegregação.

\section{CONCLUSÃO}

- O principal parâmetro que definiu o caminho de solidificação para os aços estudados foi o teor de C. O aço HTP API 5L X65 solidifica com estrutura colunar enquanto que o aço CMn_Baixo Nb solidifica com estrutura dendrítica.

- Uma maior diferença TL-Ts no aço CMn_Baixo Nb acarretou na formação de uma zona pastosa maior na frente de solidificação, com concentração mais elevada de solutos, e menor temperatura de solidificação causada pelo superresfriamento constitucional. Esta maior diferença resultou na ocorrência da linha central de segregação, claramente observada neste aço e não observada no aço HTP API 5L X65. A presença de precipitados primários de $(\mathrm{Ti}, \mathrm{Nb})(\mathrm{C}, \mathrm{N})$, maiores que $1 \mu \mathrm{m}$, e inclusões (MnS e óxidos) foi observada em todas as posições analisadas, ao contrário do aço HTP API 5L X65 onde foram encontrados somente na posição central e em menor frequência.

- Os precipitados cruciformes de $(\mathrm{Ti}, \mathrm{Nb})(\mathrm{C}, \mathrm{N})$ nuclearam em ambos os aços, ou seja, a sua precipitação é independente da estrutura de solidificação (dendrítica ou celular). O tamanho destes precipitados varia entre 100 e 500 nm. Precipitados menores que $100 \mathrm{~nm}$ apresentaram morfologia esférica.

\section{Agradecimentos}

Os autores agradecem a Companhia Brasileira de Mineração e Metalurgia-CBMM e a Gerdau Ouro Branco pelas discussões, apoio técnico e fornecimento de amostras. 
A.C.S agradece ao CNPq, CAPES e FAPERJ pelo suporte e M.S.A agradece ao CNPq pela bolsa concedida.

\section{REFERÊNCIAS}

1. Krauss G. Solidification, Segregation, and Banding in Carbon and Alloy Steels. Metallurgical and Materials Transactions B. 2003;34:781-792.

2. Lesoult G. Macro segregation in steel strands and ingots: Characterization, formation and consequences. Materials Science and Engineering A-Structural Materials Properties Microstructure and Processing. 2005;413:19-29.

3. Brune T, Kortzak K, Senk D, Reuther N, Schaperkötter M. A Three Dimensional Model to Characterize the Centerline Segregation in CC Slabs. Steel research international. 2015;86(1):33-9.

4. Zhao J, Jiang Z, Wei D. Analysis of elemental segregation in a microalloyed cast steel. Advanced Materials Research. 2013;652-654:2465-2468.

5. Pickering EJ. Macrosegregation in Steel Ingots: The applicability of Modelling and Characterization Techniques. ISIJ International. 2013;53(6):935-949.

6. Spitzig WA. Effect of sulfide inclusions morphology and pearlite banding on anisotropy of mechanical properties in normalized C-Mn steels. Metallurgical and Materials Transactions A. 1983;14(1):271-283.

7. Zhou C, Priestner R. The Evolution Solidification of Precipitates in Nb-Ti and Post-solidification Microalloyed Steels during Cooling. ISIJ International. 1996;36(11):1397-1405.

8. Palmiere EJ, Garcia CI, Deardo AJ. The influence of niobium supersaturation in austenite on the static recrystallization behavior of low carbon microalloyed steels. Metallurgical and Materials Transactions A. 1996;27:951-960.

9. Fossaert C, Rees G, Maurickx T, Bhadeshia HKDH. The effect of niobium on the hardenability of microalloyed austenite. Metallurgical and Materials Transactions A. 1995;26:21-30.

10. Rainforth WM, Black MP, Higginson RL, Palmiere EJ, Sellars CM. Precipitation of $\mathrm{NbC}$ in a model austenitic steel. Acta materialia. 2002;50:735-747.

11. Chen Z, Loretto $\mathrm{MH}$, Cochrane RC. Nature of large precipitates in titaniumcontaining HSLA steels. Materials Science and Technology. 1987;3(10):836844.

12. Lage MG, Costa e Silva ALV. Evaluating segregation in HSLA steels using computational thermodynamics. Journal of materials research and technology 2015;4(4):353-358.

13. Gray JM. Low Manganese Sour Service Linepipe Steel. In: Companhia Brasileira de Metalurgia e Mineração - CBMM. Proceedings of the Microalloyed Steels for Sour Service International Seminar; 2012; São Paulo, Brasil. Araxá: CBMM.

https://samario01.cbmm.com.br/cgs/publico/VisualizaArquivoBVPublica.ashx?D OC_Codigo $=15521$.

14. Hulka K, Bordignon P, Gray JM. Experience with low carbon HSLA steel containing 0.06 to 0.10 percent niobium: Niobium technical report, 2004, pp. 132.

15. Scheil E. Uber die eutektische kristallisation. Zeitschrift Fur Metallkunde. 1942;34:70.

16. Zhang D. Characterization and modelling of segregation in continuously cast steel slab. PhD Thesis. School of Metallurgy and Materials College of 
Engineering and Physical Sciences, University of Birmingham. September 2015.

17. Yasuhide O, Akira Y. Corrosion liquid for exposing dendrite of carbon steel or low-alloy steel cast piece, 2001, JP Patent 2001-289839A.

18. Costa e Silva ALV. Segregação em aços alta-resistência baixa liga (ARBL) para aplicações em serviço com H2S: Avaliação por termodinâmica computacional. Tecnologia em Metalurgia, Materiais e Mineração. 2014;11(1):3-13.

19. Campbell J., 2003 Second Edition, Castings, Butterworth-Heinemann, Oxford

20. Won YM., Thomas B. Simples model of microsegregation during solidifications of steels. Metallurgical and Materials Transactions A. 2001;32:1755-1767.

21. Suzuki A, Suzuki T, Nagoaka $Y$, Iwata $Y$. On secondary dendrite arm spacing in commercial carbon steels with different carbon content. Journal of JIM. 1968;9(12):1301-05.

22. Roy S, Patra S, Neogy S, Laik A, Choudhary SK, Chakrabarti D. Prediction of inhomogeneous distribution of microalloy precipitates in continuous-cast high strength, low-alloy steel slab. Metallurgical and Materials Transactions A. 2012;43:1845-1860. 